\title{
Intradural Procedural Time to Assess Technical Difficulty of Superciliary Keyhole and Pterional Approaches for Unruptured Middle Cerebral Artery Aneurysms
}

\author{
Yeon-Ju Choi, M.D., ${ }^{*}$ Wonsoo Son, M.D., ${ }^{*}$ Ki-Su Park, M.D., Jaechan Park, M.D., Ph.D. \\ Department of Neurosurgery, Research Center for Neurosurgical Robotic Systems, Kyungpook National University, Daegu, Korea
}

Objective : This study used the intradural procedural time to assess the overall technical difficulty involved in surgically clipping an unruptured middle cerebral artery (MCA) aneurysm via a pterional or superciliary approach. The clinical and radiological variables affecting the intradural procedural time were investigated, and the intradural procedural time compared between a superciliary keyhole approach and a pterional approach.

Methods : During a 5.5-year period, patients with a single MCA aneurysm were enrolled in this retrospective study. The selection criteria for a superciliary keyhole approach included : 1) maximum diameter of the unruptured MCA aneurysm <15 mm, 2) neck diameter of the MCA aneurysm $<10 \mathrm{~mm}$, and 3) aneurysm location involving the sphenoidal or horizontal segment of MCA (M1) segment and MCA bifurcation, excluding aneurysms distal to the MCA genu. Meanwhile, the control comparison group included patients with the same selection criteria as for a superciliary approach, yet who preferred a pterional approach to avoid a postoperative facial wound or due to preoperative skin trouble in the supraorbital area. To determine the variables affecting the intradural procedural time, a multiple regression analysis was performed using such data as the patient age and gender, maximum aneurysm diameter, aneurysm neck diameter, and length of the pre-aneurysm M1 segment. In addition, the intradural procedural times were compared between the superciliary and pterional patient groups, along with the other variables.

Results : A total of 160 patients underwent a superciliary $(n=124)$ or pterional $(n=36)$ approach for an unruptured MCA aneurysm. In the multiple regression analysis, an increase in the diameter of the aneurysm neck $(p<0.001)$ was identified as a statistically significant factor increasing the intradural procedural time. A Pearson correlation analysis also showed a positive correlation $(r=0.340)$ between the neck diameter and the intradural procedural time. When comparing the superciliary and pterional groups, no statistically significant between-group difference was found in terms of the intradural procedural time reflecting the technical difficulty (mean \pm standard deviation : 29.8 \pm 13.0 min versus $27.7 \pm 9.6$ min).

Conclusion : A superciliary keyhole approach can be a useful alternative to a pterional approach for an unruptured MCA aneurysm with a maximum diameter $<15 \mathrm{~mm}$ and neck diameter $<10 \mathrm{~mm}$, representing no more of a technical challenge. For both surgical approaches, the technical difficulty increases along with the neck diameter of the MCA aneurysm.

Key Words : Intracranial aneurysm $\cdot$ Middle cerebral artery $\cdot$ Pterional approach $\cdot$ Superciliary approach.

\section{INTRODUCTION}

Recent trends toward the use of minimally invasive surgery for unruptured intracranial aneurysms have been prompted by patient reluctance to surgical invasion and the availability of less invasive endovascular treatment ${ }^{12,13)}$. However, the major limitation with a minimally invasive surgical approach is the small cranial opening, incurring narrow viewing angles, limited ma- neuverability of the microinstruments, and a unidirectional surgical approach ${ }^{3,4,7,8,13-15)}$.

A superciliary keyhole approach using a supraorbital minicraniotomy has been applied as a minimally invasive alternative to a pterional approach for clipping intracranial aneurysms arising at or below the level of the circle of Willis, such as aneurysms at the supraclinoid internal carotid artery (ICA), anterior communicating artery, and middle cerebral artery (MCA $)^{13-15)}$.

• Received : February 25, 2016 • Revised : May 9, 2016 •Accepted : June 21, 2016

$*$ These authors contributed equally to this work.

- Address for reprints : Jaechan Park, M.D., Ph.D.

Department of Neurosurgery, Kyungpook National University Hospital, 130 Dongdeok-ro, Jung-gu, Daegu 41944, Korea

Tel : +82-53-420-5647, Fax : +82-53-423-0504, E-mail : jparkmd@hotmail.com

- This is an Open Access article distributed under the terms of the Creative Commons Attribution Non-Commercial License (http://creativecommons.org/licenses/by-nc/3.0) which permits unrestricted non-commercial use, distribution, and reproduction in any medium, provided the original work is properly cited. 
However, with a superciliary keyhole approach, the technical difficulty and limitations regarding the access and clipping of the aneurysm differ significantly according to the aneurysm location, and are especially critical for MCA aneurysms located deep in the periphery of the operative field.

Accordingly, this study uses the intradural procedural time to assess the overall technical difficulty of surgically clipping an unruptured MCA aneurysm via a pterional or superciliary approach. The clinical and radiological variables affecting the intradural procedural time are investigated, and the intradural procedural times compared between a superciliary keyhole approach and a pterional approach.

\section{MATERIALS AND METHODS}

\section{Patients}

During a 5.5-year period from January 2010 to June 2015, among 402 consecutive patients who underwent a pterional or superciliary approach for clipping unruptured intracranial aneurysms at the authors' institution, those patients with a single MCA aneurysm were enrolled in this retrospective study.

The selection criteria for a superciliary keyhole approach included : 1) maximum diameter of the unruptured MCA aneurysm $<15 \mathrm{~mm}, 2$ ) neck diameter of the MCA aneurysm $<10 \mathrm{~mm}$, and 3) aneurysm location involving the sphenoidal or horizontal segment of MCA (M1) segment and MCA bifurcation, excluding aneurysms distal to the MCA genu. Meanwhile, the control comparison group included patients with the same selection criteria as for a superciliary approach, yet who preferred a pterional approach to avoid a postoperative facial wound or due to preoperative skin trouble in the supraorbital area.

The patients with MCA aneurysms requiring a pterional approach or unfavorable for a superciliary approach were excluded from this study based on the following criteria : 1) aneurysm with a maximum diameter $\geq 15 \mathrm{~mm}$ or neck diameter $\geq 10 \mathrm{~mm}$ $(n=5), 2)$ aneurysm distal to the MCA genu ( $n=3)$, and 3) two adjacent aneurysms $(n=1)$ requiring a large craniotomy to allow clip application in different directions.

As the intention of this study was to use the intradural procedural time to compare the technical difficulty of treating unruptured aneurysms with two different surgical approaches, cases of multiple aneurysms and a concomitant unruptured aneurysm in association with a ruptured aneurysm were also excluded in this study. This study was reviewed and approved by an institutional ethics committee.

\section{Superciliary versus pterional approach for MCA aneurysms}

The locations of a pterional craniotomy and supraorbital minicraniotomy are depicted in Fig. 1, along with the direction of the surgical microscope. In cases of a pterional craniotomy, the sylvian fissure is located in the middle of the operative field, allowing standard dissecting maneuvers using an operative mi-

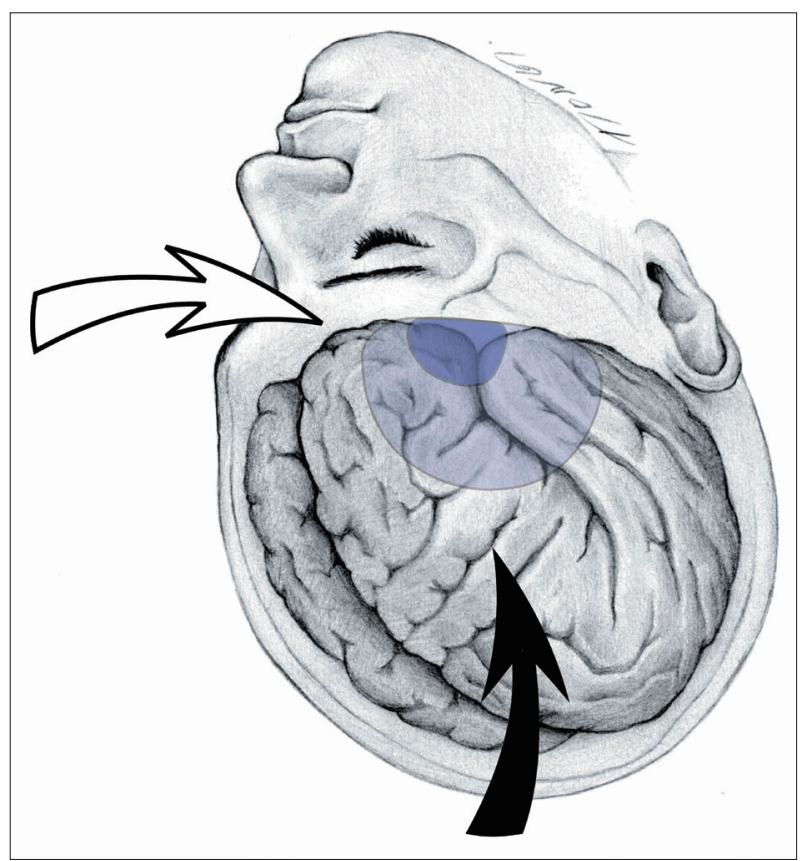

Fig. 1. Illustration showing the location of the craniotomies and microscopic direction. The surgical microscope is directed anteroinferiorly (solid arrow) with a pterional approach, while rotated and positioned in a lateral direction (empty arrow) with a superciliary approach. Note the location of the pterional craniotomy (light blue) and supraorbital mini-craniotomy (dark blue).

croscope directed anteroinferiorly.

A superciliary keyhole approach for anterior circulation aneurysms was previously described by the current authors ${ }^{9,12,13,16)}$. It comprises a $3.5 \mathrm{~cm}$ skin incision and a supraorbital mini-craniotomy with a diameter of $<2 \mathrm{~cm}$. In particular, for an MCA aneurysm, further slight $(<5 \mathrm{~mm})$ drilling of a lateral margin of the cranial opening is critical to facilitate exposure of the sylvian fissure.

The first intradural procedure is brain relaxation via the release of cerebrospinal fluid (CSF) through a small opening in the arachnoid covering the carotid cistern using an operative microscope directed anteroinferiorly. The resultant sunken brain leads to exposure of the sylvian fissure deep in the periphery of the operative field. The microscope is then rotated and positioned to achieve the maximal view toward the sylvian fissure under the lesser wing of the sphenoid (Fig. 2A). The focal $(<2 \mathrm{~cm})$ opening in the cortical arachnoid of the sylvian fissure along the superficial sylvian vein is sufficient to access most MCA aneurysms (Fig. 2B) ${ }^{6}$. Uncommonly, splitting the sphenoidal compartment of the sylvian fissure can be required for MCA aneurysms with a short $(<1.5 \mathrm{~cm})$ pre-aneurysmal M1 segment. If the sphenoidal compartment of the sylvian fissure is deviated laterally and out of view, the technique of dissection from inside out is useful.

Slender instruments, such as a dissector, tubular aneurysm clip applier, tubular microscissors, and tubular bipolar coagulator, are useful in this limited operative field. All cases of both 
approaches were conducted by the senior author.

\section{Technical difficulty}

The technical difficulty, determined by the degree of aneurysmal accessibility and instrument maneuverability, was assessed based on the intradural procedural time, which was measured using the digital recording of the intraoperative microscope video. For both approaches, pterional and superciliary, the microscope was introduced immediately after dural opening and used until aneurysm clipping. Thus, the intradural procedural time was defined as the time period from the application of the microscope after dural opening to aneurysm clipping.

\section{Technical efficacy and safety}

The technical efficacy to achieve successful clip application was evaluated based on the state of the clip placement and presence of a residual sac in postoperative computed tomography angiography (CTA), which was routinely taken on the first postoperative day.
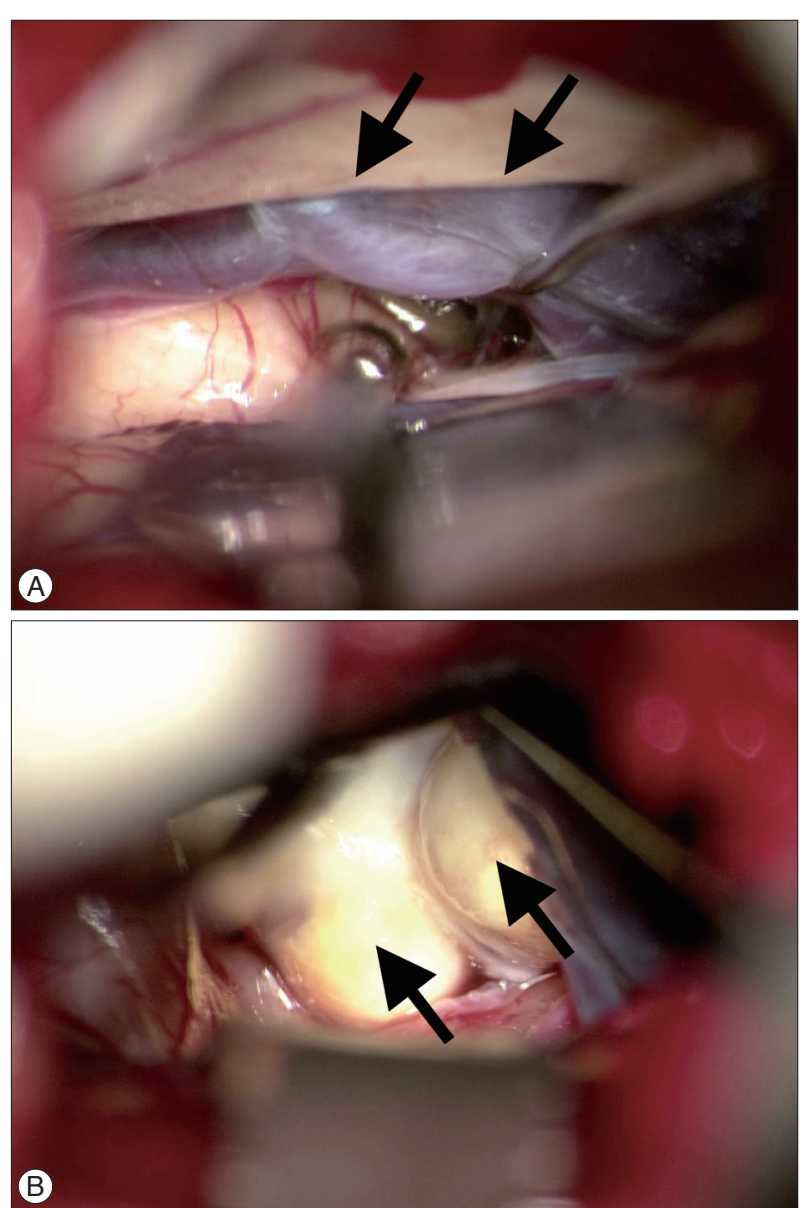

Fig. 2. Superciliary keyhole approach for an MCA aneurysm. A : Intraoperative photograph showing the sylvian fissure under the lesser wing (arrows) of the sphenoid via a superciliary approach. B : Intraoperative photograph showing an MCA aneurysm (arrows) exposed after focal opening in the cortical arachnoid of the sylvian fissure via a superciliary approach. MCA : middle cerebral artery.
Medical records and radiological data were also used to investigate the technical safety and any procedure-related complications. Thus, data were collected on intraoperative blood transfusions and postoperative complications, including an intracranial hemorrhage, cerebral infarction, intracranial or wound infection, and other postoperative morbidity.

\section{Statistical analysis}

The statistical analyses were performed with the aid of commercially available statistics software (SPSS version 19.0; SPSS, Inc., Chicago, IL, USA). To determine the variables affecting the intradural procedural time, a multiple regression analysis was performed using such data as the patient age and gender, maximum aneurysm diameter, aneurysm neck diameter, and length of the pre-aneurysm M1 segment.

In addition, the intradural procedural times were compared between the superciliary and pterional patient groups, and according to other variables, including the patient age and gender, maximum aneurysm diameter, aneurysm neck diameter, and length of the pre-aneurysm M1 segment. A two sample t-test was used for the quantitative variables, while a $\chi^{2}$ analysis was used for the categorical variables. The results were considered significant for probability values less than 0.05 .

\section{RESULTS}

\section{Patients}

A total of 160 patients with an age range from 36 to 74 years [mean \pm standard deviation (SD), 59.0 $\pm 9.2 \mathrm{yr}$ ] who underwent a superciliary $(n=124)$ or pterional $(n=36)$ approach for an unrup-

Table 1. Summary of characteristics of 160 patients who underwent surgical clipping of an unruptured MCA aneurysm

\begin{tabular}{lc}
\hline \multicolumn{1}{c}{ Characteristic } & Value \\
\hline Mean age \pm SD in yrs (range) & $59.0 \pm 9.2(36-74)$ \\
Gender (\%) & $107(66.9)$ \\
Male & $53(33.1)$ \\
Female & $5.3 \pm 2.3(3.0-14.6)$ \\
Mean aneurysm diameter \pm SD in mm (range) & $3.9 \pm 1.6(1.2-9.8)$ \\
Mean aneurysm neck diameter \pm SD in & \\
mm (range) & $18.5 \pm 5.2(5.4-30.0)$ \\
Mean length of pre-aneurysmal M1 $\pm S D$ in & \\
mm (range) & \\
Aneurysm types according to its location & $17(10.6)$ \\
Early frontal branch aneurysm & $12(7.5)$ \\
Early temporal branch aneurysm & $123(76.9)$ \\
MCA bifurcation aneurysm & $8(5.0)$ \\
MCA trifurcation aneurysm & \\
Surgical approach (\%) & $124(77.5)$ \\
Superciliary & $36(22.5)$ \\
Pterional & $29.3 \pm 12.3(7.8-59.0)$ \\
Mean intradural procedural time in min (range) & \\
\hline M1 : sphenoidal or horizontal segment of MCA, MCA : middle cerebral artery
\end{tabular}


tured MCA aneurysm were enrolled in this study. Their demographic and radiological characteristics are presented in Table 1.

On preoperative angiograms, the MCA aneurysms included an early frontal branch aneurysm $(n=17)$, early temporal branch aneurysm $(n=12)$, bifurcation aneurysm $(n=123)$, and trifurcation aneurysm $(n=8)$. The length of the pre-aneurysmal M1 segment ranged from $5.4 \mathrm{~mm}$ to $30.0 \mathrm{~mm}$ (mean $\pm \mathrm{SD}, 18.5 \pm 5.2 \mathrm{~mm}$ ).

\section{Variables affecting intradural procedural time}

In the multiple regression analysis, an increase in the diameter of the aneurysm neck $(p<0.001)$ was identified as a statistically significant factor increasing the intradural procedural time. A Pearson correlation analysis also showed a positive correlation ( $\mathrm{r}=0.340)$ between the neck diameter and the intradural procedural time (Fig. 3).

\section{Comparison between superciliary and pterional groups}

The demographic data, angiographic data, and intradural procedural times for the superciliary and pterional patient groups are summarized in Table 2 . The superciliary and pterional groups were well matched without significant difference for such variables as patient age (mean \pm SD : $58.4 \pm 9.2$ years versus $61.5 \pm 9.0$ years, $p=0.071$ ), gender (male patients : $65.3 \%$ versus

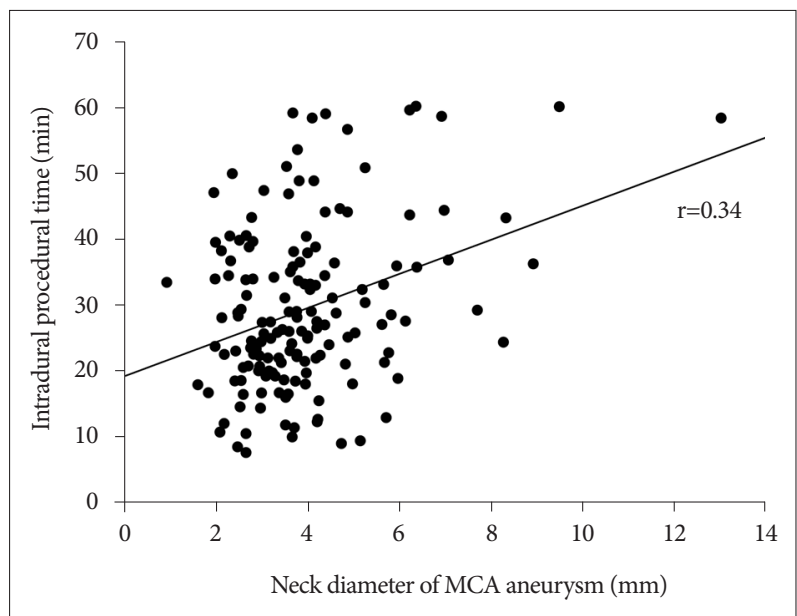

Fig. 3. Graph of Pearson correlation analysis results, showing a positive correlation ( $r=0.34$ ) between the neck diameter of the MCA aneurysm and the intradural procedural time. MCA : middle cerebral artery.
$72.2 \%, p=0.442)$, maximum diameter of the MCA aneurysm (5.1 $\pm 2.1 \mathrm{~mm}$ versus $6.0 \pm 2.6 \mathrm{~mm}, p=0.051)$, neck diameter of the MCA aneurysm ( $3.9 \pm 1.7 \mathrm{~mm}$ versus $4.2 \pm 1.4 \mathrm{~mm}, p=0.322)$, and length of the pre-aneurysmal M1 segment $(18.2 \pm 5.4 \mathrm{~mm}$ versus $19.4 \pm 4.3 \mathrm{~mm}, p=0.216$ ).

As regards the intradural procedural time reflecting the technical difficulty, no statistically significant between-group difference was identified (mean \pm SD : $29.8 \pm 13.0$ min versus $27.7 \pm 9.6 \mathrm{~min}$ ).

\section{Technical efficacy and safety : superciliary group versus pterional group}

All the MCA aneurysms in the present series were successfully clipped via a superciliary or pterional approach. No case of a residual sac was found in the postoperative CTA.

The procedure-related complications are summarized in Table 3. The pterional group experienced an epidural hematoma $>10 \mathrm{~mL}(\mathrm{n}=2)$, subdural hematoma $>10 \mathrm{~mL}(\mathrm{n}=1)$, and wound dehiscence $(n=1)$, where the epidural and subdural hematomas were asymptomatic and managed conservatively. Meanwhile, the superciliary group did not experience any epidural or subdural hematomas, although there was one asymptomatic ve-

Table 3. Procedure-related complications following the clipping of an unruptured MCA aneurysm in the superciliary and pterional patient groups $^{*}$

\begin{tabular}{|c|c|c|}
\hline Complication & $\begin{array}{c}\text { Superciliary group } \\
(\mathrm{n}=124)\end{array}$ & $\begin{array}{c}\text { Pterional group } \\
(\mathrm{n}=36)\end{array}$ \\
\hline Epidural hematoma $>10 \mathrm{~mL}$ & 0 & $\begin{array}{l}2(5.6), \\
\text { asymptomatic }\end{array}$ \\
\hline Subdural hematoma $>10 \mathrm{~mL}$ & 0 & $\begin{array}{l}1(2.8), \\
\text { asymptomatic }\end{array}$ \\
\hline Arterial infarction & 0 & 0 \\
\hline Venous infarction & $\begin{array}{l}1(0.8), \\
\text { asymptomatic }\end{array}$ & 0 \\
\hline CSF infection & 0 & 0 \\
\hline Wound infection & 0 & 0 \\
\hline Wound dehescence & 0 & $1(2.8)$ \\
\hline Permanent palsy of frontalis & 0 & 0 \\
\hline Neurological deficit & 0 & 0 \\
\hline Mortality & 0 & 0 \\
\hline
\end{tabular}

*Values are number of patients (\%) unless noted otherwise. CSF : cerebrospinal fluid, MCA : middle cerebral artery

Table 2. Comparison of patients who underwent a superciliary approach or pterional approach for an unruptured MCA aneurysm

\begin{tabular}{lccc}
\hline \multicolumn{1}{c}{ Variable } & Superciliary group $(\mathrm{n}=124)$ & Pterional froup $(\mathrm{n}=36)$ & $p$ value \\
\hline Gender (\%) & & $26(72.2)$ & $0.442^{*}$ \\
$\quad$ Male & $81(65.3)$ & $10(27.8)$ & \\
$\quad$ Female & $43(34.7)$ & $61.5 \pm 9.0(42-73)$ & $0.071^{\dagger}$ \\
Mean age \pm SD in yrs (range) & $58.4 \pm 9.2(36-74)$ & $6.0 \pm 2.6(3.0-12.5)$ & $0.051^{\dagger}$ \\
Mean diameter of MCA aneurysm $\pm S D$ in mm (range) & $5.1 \pm 2.1(3.0-14.6)$ & $4.2 \pm 1.4(1.6-7.7)$ & $0.322^{\dagger}$ \\
Mean diameter of aneurysm neck \pm SD in mm (range) & $3.9 \pm 1.7(1.2-9.8)$ & $19.4 \pm 4.3(11.7-27.9)$ & $0.216^{\dagger}$ \\
Mean length of pre-aneurysmal M1 $\pm S D$ in mm (range) & $18.2 \pm 5.4(5.4-30.0)$ & $27.7 \pm 9.6(9.5-59.0)$ & $0.382^{\dagger}$ \\
Mean intradural procedural time in min (range) & $29.8 \pm 13.0(7.8-58.0)$ & &
\end{tabular}

${ }^{*}$ Chi-square test, ${ }^{\dagger}$ Two sample t-test. M1 : sphenoidal or horizontal segment of MCA, MCA : middle cerebral artery 
nous infarct $(n=1)$. No cosmetic problems were encountered, such as permanent palsy of the frontalis muscle. In addition, neither group experienced any procedure-related permanent morbidity or mortality or procedure-related infection.

\section{DISCUSSION}

Since the introduction of the keyhole concept by Perneczky's group in $1998^{17)}$, the technical feasibility of a superciliary keyhole approach for intracranial aneurysms has already been reported in literature ${ }^{3,4,-9,12-16)}$. Moreover, the current authors previously reported on the indications and contraindications of a superciliary approach for aneurysms ${ }^{11-13)}$. Therefore, the present study investigated the level of technical difficulty produced by a superciliary approach for an MCA aneurysm based on the intradural procedural time. As a result, a superciliary keyhole approach showed the same level of difficulty as a pterional approach for clipping an aneurysm with a maximum diameter $<15 \mathrm{~mm}$ and neck diameter $<10 \mathrm{~mm}$ arising at the M1 segment and MCA bifurcation.

A superciliary approach is considered unfavorable for an MCA aneurysm in the following cases : 1) large aneurysm with a maximum diameter $\geq 15 \mathrm{~mm}, 2$ ) wide-necked aneurysm with neck diameter $\geq 10 \mathrm{~mm}, 3) \mathrm{M} 2$ aneurysm distal to the MCA genu, and 4) adjacent multiple aneurysms. Meanwhile, MCA aneurysms with a short pre-aneurysmal M1 segment are located in the sphenoidal compartment of the sylvian fissure below the sphenoid ridge, and can be more difficult to access than a MCA bifurcation aneurysm at the MCA genu. However, the length of the pre-aneurysmal M1 segment did not affect the intradural procedural time in the present series due to the usage of slender instruments and the technique of dissection from inside out.

For both approaches, superciliary and pterional, the intradural procedural time ranged widely from 10 minutes to 1 hour, although the mean time was approximately 30 minutes. In particular, the intradural procedural time increased as the neck diameter of the aneurysm increased, as a larger neck diameter requires a wider arachnoid opening along the superficial sylvian vein and more extensive deep sylvian dissection, and involves more difficult aneurysm dissection and more difficult visualization of the posterior wall of the aneurysm base to avoid compromising the perforating vessels.

The operative time for aneurysm surgery can be divided into three time periods as follows : cranial opening time (skin incision to dural opening), intradural procedural time (operative duration using an operative microscope after dural opening), and cranial closing time (dural closure to skin closure). Specifically, the intradural procedural time covers all the delicate and sophisticated procedures, including arachnoid dissection, access to the aneurysm, and aneurysm clipping, all of which are performed under microscopic view. Therefore, since this time period is directly affected by the technical limitations of a mini- mally invasive surgical approach, it can be used as a scale to indicate the technical difficulty in comparison with a conventional approach.

Meanwhile, the cranial opening time of a superciliary approach is much shorter than that of a pterional approach, as the small skin incision and creation of a supraorbital mini-craniotomy can be performed within 15 minutes. Rapid access to the intracranial ICA and MCA using a superciliary approach allows a minimally invasive and rapid surgical embolectomy for acute ischemic stroke to achieve reperfusion within the therapeutic time window ${ }^{10,11)}$.

In the present study, the intradural procedural time for an MCA aneurysm did not differ between the pterional and superciliary patient groups. However, the cranial opening time and closing time were both much shorter with a superciliary approach. Thus, the overall operative time for an MCA aneurysm was much shorter with a superciliary approach than with a pterional approach.

When compared with a standard pterional approach, the area of exposure of the parasellar region through a superciliary keyhole approach has already been quantitatively verified as adequate and similar ${ }^{2}$. In addition, surgical minimalism with tailoring the exposure to the patient's anatomy and surgical lesion has led to the application of a superciliary keyhole approach ${ }^{5}$, which offers many advantages as follows : 1$)$ small $(3.5 \mathrm{~cm})$ operative wound, 2) short operative duration due to the short cranial opening and closing time, 3) less intraoperative blood loss, 4) rarer occurrence of an epidural hematoma, 5) minimal damage to the temporalis muscle, 6) rarer occurrence of wound dehiscence or wound related pain, and 7) early return to work and normal life $\mathrm{f}^{12-14)}$.

However, as a superciliary keyhole approach is a limited procedure, it should only be considered an acceptable alternative for neurosurgeons who have gained sufficient surgical experience with the technique. The appropriate indications also need to be verified. When Chalouhi et al. ${ }^{1)}$ compared the results of pterional and superciliary keyhole approaches for ruptured aneurysms, they reported more frequent intraoperative aneurysm ruptures in the keyhole group. Thus, for the current cases, the comparable surgical outcomes and intradural procedural times for the pterional and superciliary keyhole approaches resulted from an indication of unruptured aneurysms and the surgeon's experience level. Furthermore, some patients are reluctant to undergo a facial wound, despite its small size. Therefore, the surgeon should inform the patient carefully about the advantages and disadvantages of the two surgical techniques.

The current study has several important limitations. First, it was based on a retrospective review of a small case series from a single institution. Second, the surgical results were obtained by a single experienced neurovascular surgeon, which may limit the extent to which the study results can be generalized. Third, selection bias could have occurred in this case-control study, yet was considered insignificant as the acceptance of a postopera- 
tive facial wound had more affect on the patient's selection of the surgical approach than the aneurysm characteristics.

\section{CONCLUSION}

A superciliary keyhole approach can be a useful alternative to a pterional approach for an unruptured MCA aneurysm with a maximum diameter $<15 \mathrm{~mm}$ and neck diameter $<10 \mathrm{~mm}$, representing no more of a technical challenge. For both surgical approaches, the technical difficulty increases along with the neck diameter of the MCA aneurysm.

\section{References}

1. Chalouhi N, Jabbour P, Ibrahim I, Starke RM, Younes P, El Hage G, et al. : Surgical treatment of ruptured anterior circulation aneurysms : comparison of pterional and supraorbital keyhole approaches. Neurosurgery 72 : 437-441; discussion 441-442, 2013

2. Cheng CM, Noguchi A, Dogan A, Anderson GJ, Hsu FP, McMenomey SO, et al. : Quantitative verification of the keyhole concept : a comparison of area of exposure in the parasellar region via supraorbital keyhole, frontotemporal pterional, and supraorbital approaches. J Neurosurg 118 : 264-269, 2013

3. Czirják S, Nyáry I, Futó J, Szeifert GT : Bilateral supraorbital keyhole approach for multiple aneurysms via superciliary skin incisions. Surg Neurol 57 : 314-323; discussion 323-324, 2002

4. Czirják S, Szeifert GT : Surgical experience with frontolateral keyhole craniotomy through a superciliary skin incision. Neurosurgery 48 : 145-149; discussion 149-150, 2001

5. Davies JM, Lawton MT : Advances in open microsurgery for cerebral aneurysms. Neurosurgery 74 Suppl 1 : S7-S16, 2014

6. Elsharkawy A, Niemelä M, Lehečka M, Lehto H, Jahromi BR, Goehre F, et al. : Focused opening of the sylvian fissure for microsurgical management of MCA aneurysms. Acta Neurochir (Wien) 156 : 17-25, 2014
7. Mitchell P, Vindlacheruvu RR, Mahmood K, Ashpole RD, Grivas A, Mendelow AD : Supraorbital eyebrow minicraniotomy for anterior circulation aneurysms. Surg Neurol 63 : 47-51; discussion 51, 2005

8. Paladino J, Mrak G, Miklić P, Jednacak H, Mihaljević D : The keyhole concept in aneurysm surgery--a comparative study : keyhole versus standard craniotomy. Minim Invasive Neurosurg 48 : 251-258, 2005

9. Park J : Superciliary keyhole approach for unruptured anterior circulation aneurysms : surgical technique, indications, and contraindications. J Korean Neurosurg Soc 56 : 371-374, 2014

10. Park J, Hwang YH, Huh S, Kang DH, Kim Y : Minimally invasive and rapid surgical embolectomy (MIRSE) as rescue treatment following failed endovascular recanalization for acute ischemic stroke. Acta Neurochir (Wien) 156 : 2041-2049; discussion 2049, 2014

11. Park J, Hwang YH, Kim Y : Extended superciliary approach for middle cerebral artery embolectomy after unsuccessful endovascular recanalization therapy : technical note. Neurosurgery 65 : E1191-E1194; discussion E1194, 2009

12. Park J, Kang DH, Chun BY : Superciliary keyhole surgery for unruptured posterior communicating artery aneurysms with oculomotor nerve palsy : maximizing symptomatic resolution and minimizing surgical invasiveness. J Neurosurg 115 : 700-706, 2011

13. Park J, Woo H, Kang DH, Sung JK, Kim Y : Superciliary keyhole approach for small unruptured aneurysms in anterior cerebral circulation. Neurosurgery 68 (2 Suppl Operative) : 300-309; discussion 309, 2011

14. Reisch R, Perneczky A : Ten-year experience with the supraorbital subfrontal approach through an eyebrow skin incision. Neurosurgery 57 (4 Suppl) : 242-255; discussion 242-255, 2005

15. Reisch R, Stadie A, Kockro RA, Hopf N : The keyhole concept in neurosurgery. World Neurosurg 79 (2 Suppl) : S17.e9-.e13, 2013

16. Shin D, Park J : Unruptured supraclinoid internal carotid artery aneurysm surgery : superciliary keyhole approach versus pterional approach. J Korean Neurosurg Soc 52 : 306-311, 2012

17. van Lindert E, Perneczky A, Fries G, Pierangeli E : The supraorbital keyhole approach to supratentorial aneurysms : concept and technique. Surg Neurol 49 : 481-489; discussion 489-490, 1998 